\title{
Institutional Obstacles and Suggestions about Implementation of Chinese Tiered Medical Pattern
}

\author{
Shanshan Li \\ School of Economics \\ Sichuan University \\ Chengdu, China \\ Jining medical university \\ Jining, China
}

\author{
Xia Lei \\ School of Economics \\ Sichuan university \\ Chengdu, China

\section{Xin Li} \\ Jining medical university \\ Jining, China
}

\author{
Fanghui Hu* \\ Jining medical university \\ Jining, China \\ *Corresponding author \\ Ping Shi \\ Jining medical university \\ Jining, China
}

\begin{abstract}
Tiered medical pattern which contains primary diagnosis, two-way referral, separate treatment of acute and chronic illnesses, operation between upper and lower medical organizations depends on smooth operation and coordination of medical management system. By analyzing current relative system in China, it is assumed that the main reasons for the difficulty in establishing the tiered medical system are that administrative hierarchy system leads excellent medical resources to aggregate in higher level hospital, two-way referral system is not perfect, and good medical culture has not yet formed. Therefore, classified medical care mode must be realized through reforming the salary system, management system, referral system, and the cultivation of reasonable medical treatment culture.
\end{abstract}

Keywords - classified medical care mode; primary diagnosis; referral system; institutional obstacles; resource allocation

\section{INTRODUCTION}

Tiered medical system is considered as an important measure that can allocate medical resource, and promote the equalization of basic medical and health service. More than ten years have past since the Ministry of Health of the People's Republic of China proposed classification of diagnosis and treatment in 2003; after some pilot areas, it has been promoted all over the country. However, the performance is not as expected. According to the data from some medical institutions, hospital visits grow faster than grassroots organization, and hospital bed utilization rate is higher than the community. [1]-[2] It hasn't formed good medical order. The basic medical and health services are still poor, so do the accessibility and fairness. The co-existence of

The paper is sponsored by Science and Technology Agency in Shandong Province (2016RKB14040), Jining medical university (JY2016RW005) waste and shortage of resources is still serious. Apparently, the current difficulties of Tiered medical mode mainly lies in two aspects: firstly, good doctors are not willing to work in primary medical institutions; patients do not trust primary care and are not willing to go to grass-roots medical institutions, which due to primary medical institutions cannot play the role of gatekeeper. Secondly, two-way referral operates poorly; cooperation mechanism at all levels of hospitals is too difficult to achieve; the essence of it is improper or even no institutional arrangements - the way of administrative allocation of resources makes the primary medical institutions lack of resources with high quality, Two-way referral system is not perfect so that it is difficult for hospitals to work together and take patient's interest into consideration. Tiered medical system cannot achieve treatment mode which is divided on the surface, but combined in practice.

\section{ANALYSIS OF INSTITUTIONAL BARRIERS IN} CONSTRUCTING GRADING DIAGNOSIS AND TREATMENT

\section{A. Taking Administrative Way of Resources Allocation as the Main Body Is Bad for Downflow of Excellent Medical Resources}

1) Administrative leveled system leading excellent medical resources to accumulate to higher levels: At present, public organizations dominate the medical service market. The medical organizations are divided into three classes via administrative measures such as regional health planning and so on. Public resources are distributed in strict compliance with the administrative levels. The higher the 
administrative rank of the medical organization is, the more prominent the hardware devices, human resource, subject development and research are. It will push excellent doctor resources forward, and the medical skills of doctors in class three hospitals are higher than that in class two hospitals, which are higher than that in communities. The Primary doctors' skills are the lowest, so do their salaries and other disposable resources. According to the Statistical Data of Chinese Health and Family Planning in 2015, devices costing over 10000 Yuan in hospitals are 6.99 times of in community hospitals, and beds in hospitals are 3.07 times of those in community hospitals. The higher the level of the hospital is, the more the doctors with high degrees and technical titles are. Administrative allocation of resources results in unreasonable medical supplies, which causes medical resources to accumulate in higher medical organizations and to be lack in lower ones. Therefore, patients' demands are distorted that numerous patients want to go to higher-level medical organizations.

2) Management system of primary medical organizations is bad for retaining staff: Besides the disadvantage in improper allocation of resources, primary medical systems lack mechanism of talents attraction. Since 2008, primary medical organizations have been authorized the title of public insititutions again, and implement the policy of national essential medicines, two lines of revenue and expenditure, zero margin drug profit and so on. The purpose of it is to change the phenomena of increasing hospital revenue from excessive sales of drugs, and recover public welfare and lower patients' expenditure. However, the average allocation system and financial management of two lines of revenue and expenditure stifle enterprise and initiative of primary doctors. The essential medicine system limits the diagnosis of primary medical staff, reducing the diagnosis willingness of patients. At the meantime, primary medical staff undertakes the task of local public health. Heavy work exhausted the medical staff so much that they will not make efforts to studying to improve their skills. On condition of it, it becomes difficult for primary medical organizations to attract and cultivate excellent doctors with rich clinical experience.

3) Personnel management system is bad for downflow of excellent doctors: Doctors with staffing of government affiliated institutions in public hospitals, posse a lifelong job and various benefits such as standard of doctors' income accounting, promotion of technical titles, elderly support, and training. Distributing resources based on administrative class makes it possible that an increasing number of excellent talents pour into the higher-class hospitals. Rare multi-site practices is a typical example. Although multi-site medical practitioner will probably improve their income, obstacles in technical titles promotion, further training and scientific research greatly reduce doctors' passion about multi-site practice. Even though some individual doctors want to leave public hospitals and practice freely, they will face more complicated examination, approval procedures and higher entry barrier.

\section{B. Unperfect Two-way Referral System}

- Disorder of different level hospitals function is not conducive to carrying out the grading health care system. Clear position of medical institutions is the precondition of two-way referral. At present, all tiered medical institutions in China mostly apply to the administrative level. According to the hospital factors such as functions, facilities, technical strength, most of the medical institutions set up general practice and specialty medicine. Service items and scope of all tiered hospitals are consequently repeated and crossed. Community hospitals diagnose and treat the special disease, meanwhile high-level hospitals also recognize, diagnose general diseases, thus hindering the development of Tiered medical system.

- The competition mechanism between medical institutions hinders the implementation of two-way referral. In foreign countries, referral occurs mainly between general practitioners and specialists, but in China, it occurs between medical institution. ${ }^{[3]}$ Since the marketing reform in the 1980s, medical institutions in China has been operated by marketoriented self-sustaining manner, which has pursued the economic benefit maximization. ${ }^{[4]}$ Therefore the mechanism of division of labor and cooperation between medical institutions is replaced by competition mechanism. Medical institutions make decisions in the referral process from their own economic interests rather than the interests of patients to maximize their own benefits.

- There is no standard for referral. As the disease itself has a great deal of uncertainty and complexity, the classification of disease is the key point and difficulty of grading health care system. From consultation to policy documents in the pilot areas, we don't find the specific grading of the disease and treatment subjects, nor the operational implementation directories and guidelines. The policy for graded referral is unclear, and the referral between medical institutions at all levels is lack of standards. It is also lack of institutional constraints on that the referral depends entirely on the doctor's subjective judgment.

\section{Lacking of Good Medical Culture}

Economy is the foundation of the formation of hospital culture. On the one hand, China's resident income significantly increased since the reform and opening, people have higher requirements on the quantity and quality of health care, and they are willing to make higher expenditure in exchange of health. On the other hand, the reality of the medical resources gradually flowing upward to higher medical institutions leads to mistrust primary medical institutions. Besides, patients don't understand the relative professional medical information enough, nor make clear about the purpose and process of the grading health care system, which leads to choose medicine institutions 
disorderly. A survey from Wang Yali shows, 55.10\% of the people do not understand the grading health care system, and $61.20 \%$ of them do not accept the system.[5] In order to avoid unnecessary risks, they often choose large-scale medical institutions to obtain higher quality and more comprehensive medical services.

\section{COUNTERMEASURES FOR THE CONSTRUCTION OF TIERED MEDICAL SYSTEM}

\section{A. Reforming the Resource Allocation Mode Centered on Administrative Allocation}

1) Reforming the current salary system and personnel administration system: Basic-level medical organizations should abolish the separation system between revenue and expenditure, allowing the subordinate community medical organizations to be more independent in employment and allocation. The personnel system and allocation system shall be established to enable the medical workers to be freer to hunt a job, take both a higher post and a lower one, and gain corresponding pay for hard work, which could further promote the initiative of medical workers in the community. Through the reform of the personnel management system, high-level medical organizations shall abolish the current manning quotas system and the fringe preferential policies, so that the excellent medical workers could become true freelancers who could choose their workplace freely. Doctors could work in hospitals in various levels voluntarily according to their own specialties and merits, which is instrumental in realizing the maximization of personal benefits and optimum of social resources allocation.

2) Establishing competitive initial medical care system: Requirements for starting medical business shall be lowered and private clinics shall be encouraged, so that basic-level medical organizations could be able to compete with each other positively. Residents shall have more chances to sign contracts with clinics independently. Meanwhile, the government-led basic medical insurance shall cover the consumption of medicine and services with nature of public commodity in the community clinics in the form of payment according to registered headcount. Financial subsidies such as funding for public health shall adopt the resident-oriented modes of government purchasing. Besides, the liberal profession for doctors shall be guaranteed with professional liability insurance.

\section{B. Improving the Two-way Referral System}

1) Clarifying the function orientation of hospitals in different levels: The function of hospitals in hierarchical diagnosis and treatment shall be classified according to different features of general medicine and special medicine, but not their administrative ranks. To be specific, primary medical institutions, as the starting point and ending point of hierarchical diagnosis and treatment, shall be established basing on institutions with proficient in general practice. The primary medical institutions are responsible for basic medical care service by establishing close and reliable doctor-patient relationship. The secondary and tertiary medical institutions are institutions with specialist doctors. The difference between the secondary and tertiary medical institutions only lies in the difficulty in treatment of diseases. The secondary hospitals deal with common diseases and frequently-occurring diseases, while the tertiary ones should be in charge of the treatment of acute and severe diseases and complex diseases, and relevant teaching and research.

2) Transforming competition among medical institutions into division of labor and cooperation: The barriers of hierarchical and localized management system characterized with segregation of hospitals at different levels shall be removed. And medical institutions shall organize and form a medical group voluntarily, introducing and applying new management and operation mechanism in such forms as joint-stock system, joint-stock cooperative system, single proprietorship, joint venture, medical business with social funds, state-owned and non-governmental running or even private business. In this way, the management system, operational mechanism and efficiency of hospitals could be improved to further realize the optimization of profits, service and identity of a common goal by removing differences, and form a new pattern characterized by interior cooperation and exterior competition.

3) Developing a complete and integrated two-way referral system in terms of actual situation: Firstly, developing a complete and integrated two-way referral system in terms of actual situation from the perspective of medical science by medical management institutions to clarify the principles, standards and procedures for transfer treatment among hospitals in both the same level and different levels, simplify two-way referral procedures, and provide conditions for transfer treatment of patients. Secondly, guaranteeing the implementation of two-way referral with medical insurance system. On the one hand, supervision shall be strengthened on the transfer treatment of hospitals by medical insurance institutions. For example,it should make rules that the medicare payment proportion shall be reduced and the hospitals of medical insurance shall be cancelled, in case that qualified doctors and medical institutions do not implement transfer treatment as required, so as to constrain doctors and stimulate hospitals to strengthen referral management. On the other hand, transfer treatment according to formal procedures shall be encouraged by allowing patients larger proportion of medical reimbursement, more efficient hospitalizing procedures, and regulating the starting line of payment for each transfer treatment, etc.

\section{Cultivating a Favorable Health Seeking Culture}

The cultivation of favorable health seeking culture depends on, on the one hand, the improvement of service quality in basic-level medical institutions to strengthen the faith and identity of residents in these institutions, and on the other hand, the policy publicity of both hierarchical 
diagnosis and treatment and health education. We shall reform the allocation of medical resources and introduce competitive mechanism to promote service quality of basiclevel medical institutions. The communities shall help residents form rational health values and health seeking values by publicizing regularly the methods about disease prevention and self-diagnosis, and the function and specialties of hospitals at different levels, which could enhance the health awareness of residents. The publicity of hierarchical diagnosis and treatment system shall be strengthened to enable it to find its way deep into the heart of people. Mass media and new media such as internet, television, WeChat and so on shall be applied to the form of feature programs, lectures and news reports to introduce the detailed distribution of local medical resources, referral procedures and standards, and medical treatment charges and proportion of reimbursement of hospitals at different levels, as well as the approach of them, so that the patients shall be clearer about the procedures and their benefits can be better safeguarded.

\section{CONCLUSION}

From the medical point of view, the nature of tiered medicine is division of labor and collaboration between general practice and special care. Division of labor is to play good function of medical institutions, and implement reasonable matching of treatment and disease; collaboration reflects the essence of taking patients as the center, with the goal of safeguarding the health of the residents. General practice is the basis and key point of tiered medicine. From the standpoint of the allocation of resources, it is the reasonable configuration of elements in all levels of medical institutions, such as doctors, equipment, etc. From the system point of view, it is the results of smooth operation and coordination of medical management system. Both division of labor and collaboration of general practice and special care and efficient allocation of resources depend on reasonable system. Therefore, the system construction is the base and key of tiered medical pattern.

\section{REFERENCES}

[1] The national health and family planning commission of the People's Republic of China. Statistical bulletin health and family planning career development in our country in 2014. http://www.moh.gov.cn/guihuaxxs/s10742/201511/191ab1d8c5f240e 8b2f5c81524e80f19.shtml.

[2] The national health and family planning commission of the Ppeople's Republic of China. the national health service in 2015. http://www.nhfpc.gov.cn/mohwsbwstjxxzx/s7967/201512/064703111 8294696a5abf030955ef6ff.shtml.

[3] Jian 1v. "Improvement of Hierarchical Diagnosis and Treatment System Under Deepening Medical and Health Reform", Chinese Hospital Management, VOL.34, PP.1-3, June, 2014.

[4] Yincai Li. "Tiered Medical System Reform of Formation Mechanism in Perspective of Institutional Framework ". Modern Economic Research.Jiangsu, vol.403, pp.53-57, July 2015.

[5] Liya Wang." An Investigation of Cognition Situation of Hierarchical Medical System by Civilian". Chinese Health Service Management. Chengdu, vol.32, pp.423-425, June 2015.
[6] Hengpeng Zhu." Tiered Medical System Killed by Administrative Controls". Health Management. Wulumuqi, vol.49, pp.53-57, April 2014. 